\title{
Two low-cost digital camera-based platforms for quantitative creatinine analysis in urine
}

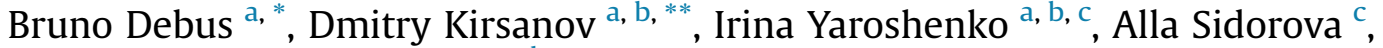 \\ Alena Piven ${ }^{\mathrm{c}}$, Andrey Legin ${ }^{\mathrm{a}, \mathrm{b}}$ \\ a Institute of Chemistry, St. Petersburg State University, St. Petersburg 199034, Russia \\ b Laboratory of Artificial Sensory Systems, ITMO University, St. Petersburg 197101, Russia \\ c Bioanalytical Laboratory CSU “Analytical Spectrometry”, St. Petersburg State Polytechnical University, St. Petersburg 198220, Russia
}

\section{H I G H L I G H T S}

- Description of two homemade platforms for the cheap quantification of creatinine level in urine.

- Image processing to extract absorption spectra and spectral fingerprints.

- PLS regression for creatinine evaluation in urine from digital camera images.

- Validation of results with capillary electrophoresis reference data.

\section{A R T I C L E I N F O}

\section{Article history:}

Received 13 July 2015

Received in revised form

31 August 2015

Accepted 1 September 2015

Available online 10 September 2015

\section{Keywords:}

Creatinine

Chemometrics

Digital camera

Spectroscopy

\section{G R A P H I C A L A B S T R A C T}

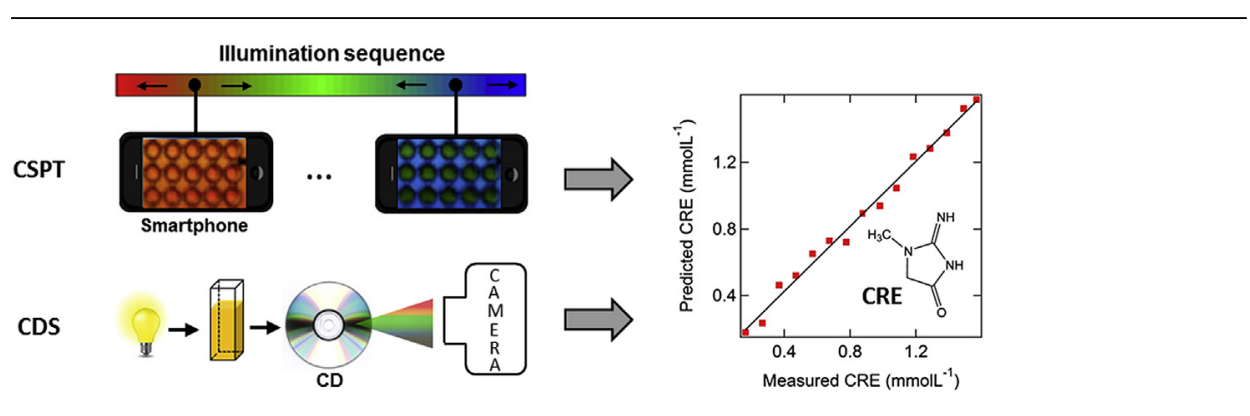


damage [1]. The variation in creatinine level in blood and urine is an important parameter in clinical diagnostics. Normal creatinine levels in urine are in the range from 3.6 to $27 \mathrm{mmol} \mathrm{L}^{-1}$ and from 3.3 to $22.5 \mathrm{mmol} \mathrm{L}^{-1}$ for men and women, respectively [2]. Abnormal level can indicate either renal malfunction in situation where creatinine concentration increases or can be a sign of muscular dystrophy for lower creatinine values. Due to its medical relevance the development of a fast and accurate assay for the determination of creatinine in human serum or urine is required.

In recent literature several techniques have been reported for creatinine assay such as HPLC [3], potentiometric biosensors [4,5], electro-analytical [2,6] and enzymatic [7] based methods, tandem mass spectroscopy [8-11], microchip capillary electrophoresis [12-14], strip assay [15,16] and Raman scattering spectroscopy [17]. Typical standard methods for creatinine quantification in urine samples are high performance liquid chromatography (HPLC) and capillary electrophoresis. Despite the broad range of available techniques for the determination of creatinine, it should be noticed that most of these approaches either require long sample preparation, tailored engineering design, skilled operators or expensive devices. Other limitations include the bulkiness of measurement systems as well as their power supply issue which both restrict creatinine assay to the specialized laboratory scale. The development of alternative inexpensive and fast creatinine quantification techniques which can be potentially applied on-site in distant locations without qualified laboratory personal is a very attractive idea.

A method, which used to be a standard in clinical laboratories before, is based on the classic colorimetric Jaffe reaction [18]. The latter involves the formation of the orange-yellow (in web version) Janovsky complex (Fig. 1), which can be monitored at $500 \mathrm{~nm}$ by spectrophotometry, following the reaction between picric acid and creatinine under alkaline conditions [19]. Although simple to implement, this reaction suffers from a lack of selectivity regarding creatinine since other metabolites of the sample can form colored complexes while reacting with alkaline picrate [20]. In practice, the negative influence of chemical interferents can be minimized by using strong alkaline medium [6] and specific temperature conditions [20]. Another way around is the two-point kinetic assay which is based on the observation that non-creatinine chromogens usually have a lower rate reaction than creatinine [21]. Thus, the color development evaluated over the first moments of the kinetic reaction can efficiently reduce the negative impact of chemical interferents.

Recently a lot of efforts have been dedicated to develop cheap and portable devices for the determination of clinically relevant metabolites in urine [22-24], blood [25,26] and others body fluids such as sweat and saliva [27]. Here cost-saving is achieved by using functionalized papers which display a color variation proportional to the concentration of analyte in the sample. To avoid subjective interpretation of the color, human eye is substituted for smartphone high resolution built-in camera, used as a colorimetric sensor [28]. A digital image of the colorimetric paper is acquired by the camera and can be either analyzed directly or transferred to a healthcare facility via a network platform for further processing $[22,29]$. Basically, the paper color is described as a linear combination of three filters defining the red, green and blue channels (RGB) of the camera. These coordinates are then compared to a predefined calibration curve to evaluate the analyte concentration. Digital scanners or cameras are also promising candidates to record changes in optical properties of the sample. In practice the sample array is illuminated by a sequential range of predefined wavelengths obtained by blending the three primary colors of a monitor screen. The light transmitted through the sample is then recorded with a camera or a webcam and converted into a spectral fingerprint. This method, known as the Computer Screen Photo-Assisted Technology (CSPT) [30], enables the simultaneous evaluation of absorbance and fluorescence of the sample. Advantages from the different systems listed above are manifold as they are inexpensive, portable and do not require specific equipment or skilled operator.

In this paper we describe two simple, cost-effective platforms for quantitative determination of creatinine in human urine: $i$ ) a CSPT array with a smartphone-based detection and ii) a homemade CD-Spectroscope (CDS) coupled with a digital camera. Pictures obtained from these devices are converted into absorption spectra (CDS) and spectral fingerprints (CSPT) by home-made procedures and analyzed with Partial Least-Squares (PLS) [31] regression to predict the concentration of analyte in the sample. The performance of both systems was evaluated with the set of 26 real human urine samples and a good agreement with benchmarked capillary electrophoresis data was established.

\section{Material and methods}

\subsection{Chemicals, reagents and samples}

All chemicals and reagents were of analytical grade. Sodium hydroxide and picric acid were purchased from Eco-Analytika and Vekton (St. Petersburg, Russia), respectively. Standard creatinine (99\%) was supplied by Acros (Gell, Belgium).

Reagents for the Jaffe reaction were prepared daily by mixing aqueous $9 \mathrm{mmol} \mathrm{L}^{-1}$ picric acid and $0.4 \mathrm{~mol} \mathrm{~L}^{-1}$ sodium hydroxide solutions at the $1: 1$ volume ratio. Working standard solutions $(\mathrm{n}=15)$ in the range from $160 \mu \mathrm{mol} \mathrm{L} \mathrm{L}^{-1}$ to $1.6 \mathrm{mmol} \mathrm{L}^{-1}$ were prepared by dilution from a fresh stock solution at $22 \mathrm{mmol} \mathrm{L}^{-1}$ creatinine in distilled water.

26 urine samples collected from healthy volunteers were provided by the Urolithiasis Laboratory (Medical Center of Laboratory Diagnostics, St. Petersburg). All samples (50 mL each) were stored in a biomedical fridge at $-25{ }^{\circ} \mathrm{C}$ until the measurement. Prior

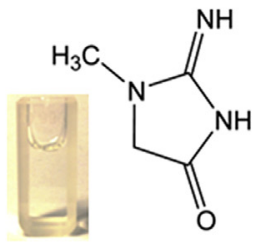

Creatinine

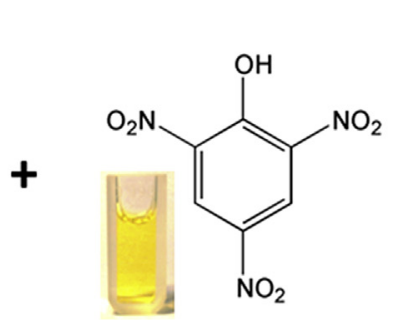

Picric acid

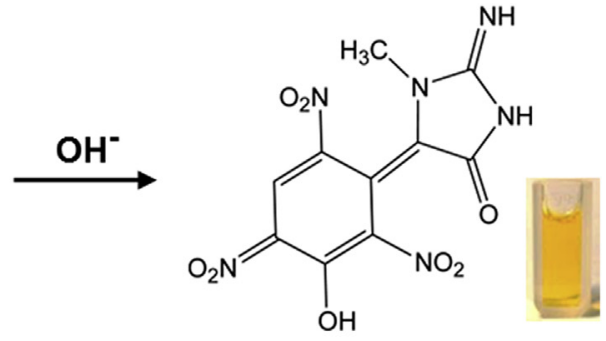

Janovsky complex

Fig. 1. Reaction scheme for the Jaffe reaction. 
analysis, samples were thawed on a water bath at room temperature $\left(25^{\circ} \mathrm{C}\right)$ and $80 \mu \mathrm{L}$ of urine were diluted with $920 \mu \mathrm{L}$ of distilled water.

\subsection{Reference data on urine samples}

The content of creatinine was determined with the capillary electrophoresis system Agilent 1100 CE (Agilent, USA) equipped with diode array detector (190-600 nm). Measurements were performed in the Bioanalytical Laboratory CSU "Analytical Spectrometry" (St. Petersburg, Russia) following the procedure described earlier [32] and the results were used as reference data. The concentration of creatinine in real human urine samples was determined to be in the range 4.44-18.73 $\mathrm{mmolL}^{-1}$.

\subsection{Development of the CD-Spectroscope (CDS)}

In order to monitor the change of absorbance of the colored Janovsky complex at $500 \mathrm{~nm}$, optical devices such as prisms or gratings are required to select the proper wavelength of interest. In the case of gratings, light dispersion is enabled by either equallyspaced parallel slits (transmission grating) or reflective stripes (reflective grating). Even if such devices can be purchased at a reasonable cost, they can be easily substituted with cheaper and more abundant materials found in everyday life. Among them compact discs are promising candidates considering that their recording tracks are spaced by $1.6 \mu \mathrm{m}$ (625 lines $/ \mathrm{mm}$ ), which served as a reflective grating. Although compact disc spectroscopes are quite popular in education to stimulate student's interest in color and light [33], their utilization for the purposes of quantitative analysis have not been reported so far, to the best of our knowledge.

The design of the home-made CD-Spectroscope is shown in Fig. 2. The spectroscope body can be typically made from any existing opaque card boxes. To reduce the size of the spectroscope and make it easy to build, a $1 \mathrm{~L}$ juice carton was used in the present study. Both top and bottom side of the carton were removed and the inner part was covered with black card paper to avoid light reflection. The carton was then sealed on each extremity by flat opaque card to prevent light from entering the box. To capture the light from the front of the device, a $1 \times 3 \mathrm{~cm}$ window was cut at one extremity and covered with two dark paper cards with parallel edges to form the adjustable slit of $3 \mathrm{~cm}$ height and $0.2 \mathrm{~mm}$ width. On the other extremity a circular "observation" window of $2.5 \mathrm{~cm}$ diameter was cut to install the reflective grating. 1/8 part of a compact disc (Verbatim), for which the reflective layer has been removed using tape, was then centered on the "observation" window and glued. The whole spectroscope dimensions are $20.5 \times 8 \times 6.5 \mathrm{~cm}(\mathrm{~L} \times \mathrm{W} \times \mathrm{H})$. The experimental setup is completed by a tungsten-filament strip desk lamp located on the edge of the CD-Spectroscope. To avoid light saturation the lamp is pointed at a blank paper sheet located $30 \mathrm{~cm}$ ahead of the slit. This layout ensures that the $1 \times 1 \times 3 \mathrm{~cm}$ glass cuvette containing the sample and placed $0.5 \mathrm{~cm}$ in front of the slit is illuminated by reflected light only. With this configuration the light transmitted through the sample is collected by the slit and moves further towards the reflective grating. A digital camera fixed on a tripod near the "observation" window completes the spectroscope to take photographs of absorption spectra of solutions. Due to its small dimensions the DCE610 model from BenQ (6.1 M pixels camera) was chosen in the present study. Captured pictures were 8-bits JPEG format with a resolution of $2112 \times 2816$ pixels. To avoid environmental light interference all experiments were carried out in the dark. Under these conditions the image quality is mainly defined by a tradeoff between exposure time and light sensitivity (ISO). Even though long exposure time can be achieved, as the camera is fixed on a tripod, setting the appropriate ISO value is not obvious. When light sensitivity increases, noise is generally introduced into the image and blurs the overall picture. In order to easily find the right balance, shooting parameters were set to automatic mode. The spectral range covered by the CD-Spectroscope is ranging from 425 to $660 \mathrm{~nm}$ which is suitable for the observation of the orange-yellow Janovsky complex at $500 \mathrm{~nm}$.

\subsection{Development of a CSPT platform}

The experimental setup is composed of the ordinary LCD computer screen (Flatron L1742SE - LG) and the smartphone (Nokia E72 with $5 \mathrm{M}$ pixels built-in camera, resolution of $480 \times 640$ pixels) used as light source and detector respectively. For CSPT measurements a Matlab (The MathWorks Ltd., Massachusetts) routine was written to display the sequence of 50 colors ranging from red to blue by blending the three primary colors (RGB). The color sequence is shown by the scattered plot in Fig. 3a (25 points out of 50 are displayed for the sake of clarity) where each color dot corresponds to the one from the illuminating sequence. The sequence was converted into the Audio Video Interleave (AVI) format displaying 2 colored frames/second for a total duration of $25 \mathrm{~s}$.

In practice, the monitor was laid down on a flat surface so that the screen was parallel to the ground. A transparent plastic 96-well microplate containing samples array (100 $\mu$ L each), was held $10 \mathrm{~cm}$

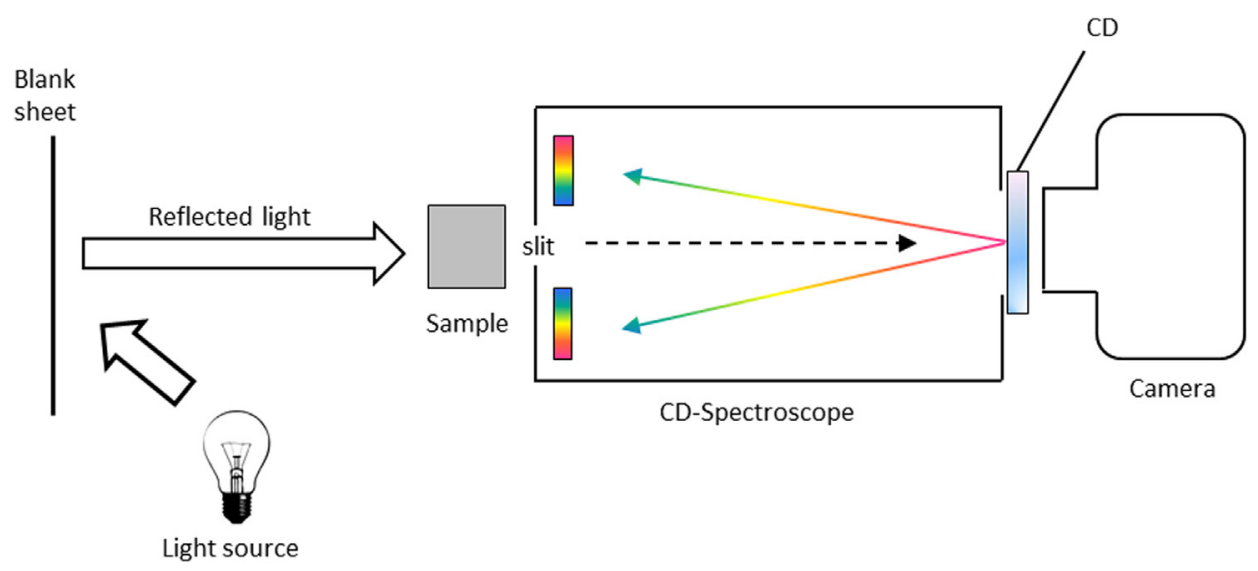

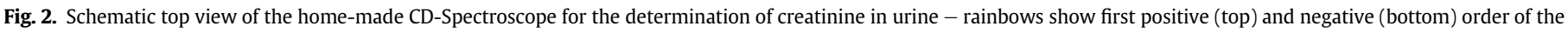
CD grating. 
a)

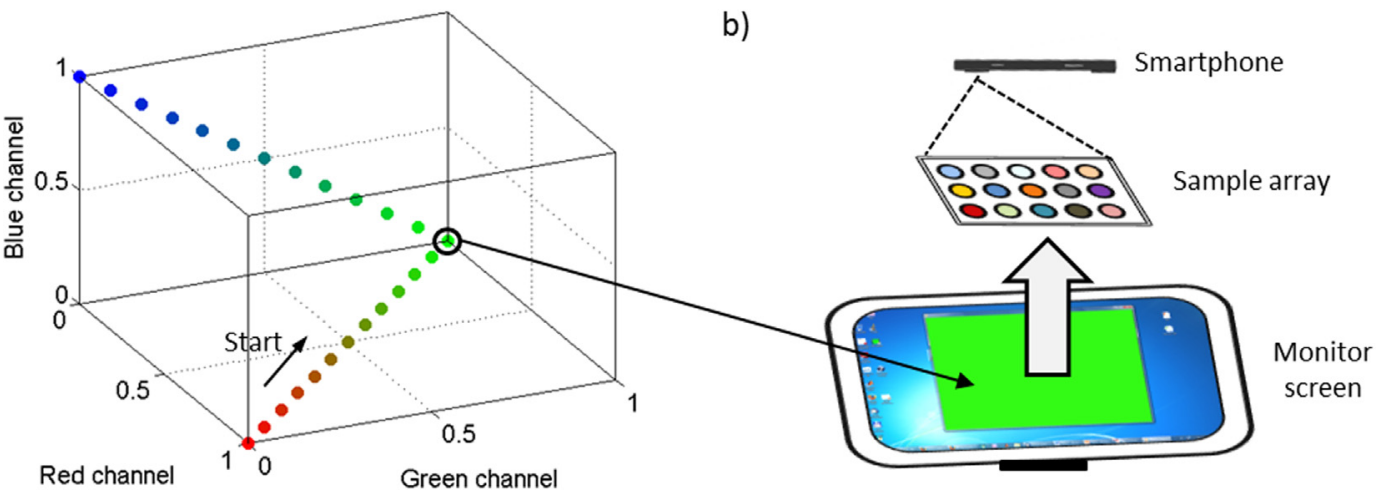

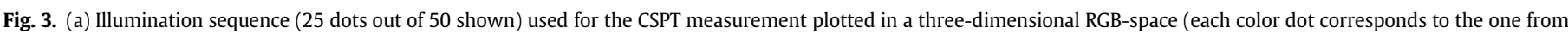

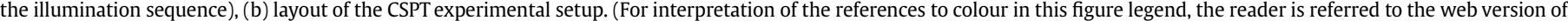
this article.)

above the screen by a laboratory stand. To capture the spectral fingerprints of the sample array during the illumination sequence, the smartphone was mounted on a laboratory clamp $18 \mathrm{~cm}$ above the well microplate. The general scheme of the experimental setup is displayed Fig. 3b. Careful examination is required prior experiment to ensure that the sample array and the smartphone are parallel in order to avoid distortion of the sensing areas of the well microplate. Experiments were performed in the dark to avoid environmental light interference.

\subsection{Image and video processing}

Multimedia data acquired by both CDS and CSPT platforms were processed in Matlab 7.5. For CDS pictures a $460 \times 120$ pixels region of interest (ROI) was selected from the first positive order of the CD grating as displayed in Fig. 4. Thus potential optical aberrations such as field curvature can be minimized since the analysis will be performed only on a subset of the raw image. Each horizontal line of the ROI contains the pixel intensity of the optical signal transmitted through the sample at a particular wavelength. The ROI was first converted into grayscale to get a single color channel and then the 120 horizontal lines were averaged to obtain a smoothed optical signal for a particular sample. Absorbance spectrum of the sample can thus be determined from Eq. (1).

Abs $=-\log \left(\frac{I}{I_{0}}\right)$

where I and $\mathrm{I}_{0}$ correspond to the intensity of the signal transmitted through the sample and the alkaline picrate solution respectively. In order to get wavelength absorbance spectra a pixel to wavelength conversion was applied by recording the spectrum of a mercury vapor fluorescent lamp under the same experimental conditions. The pixel position of the main absorption band was regressed against reference values in nanometers and the calibration line was built. This procedure will be discussed later in the
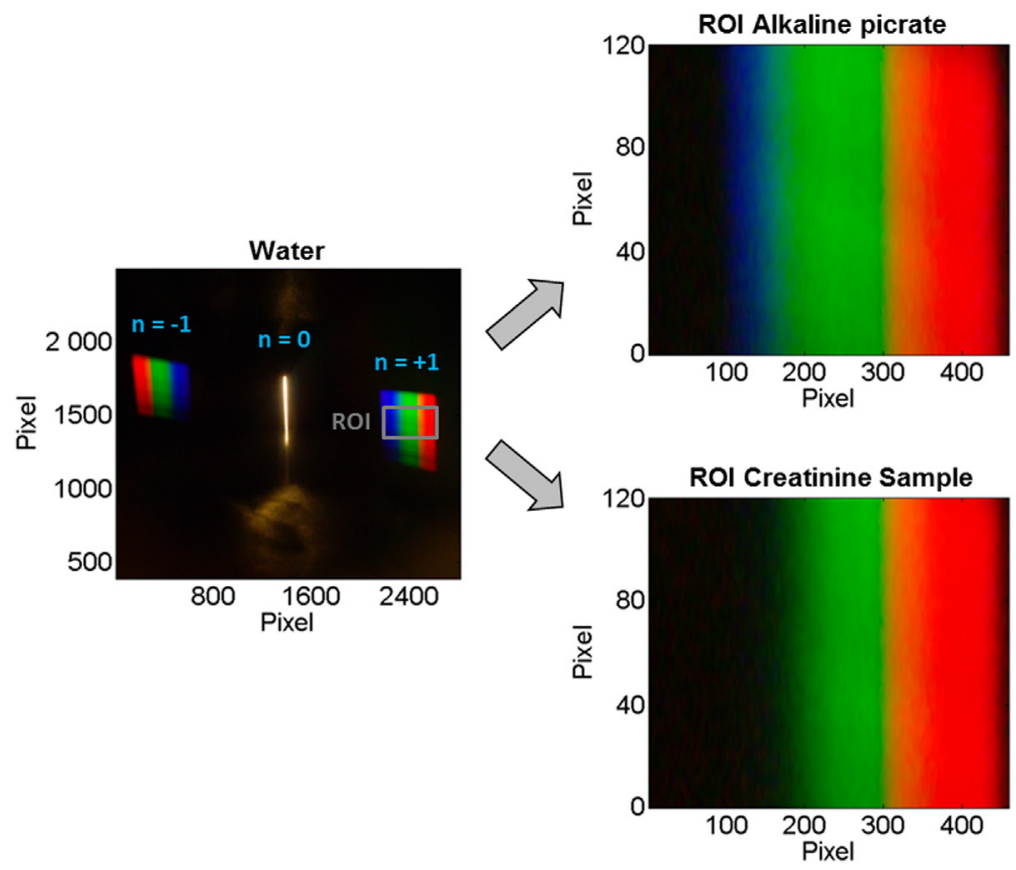
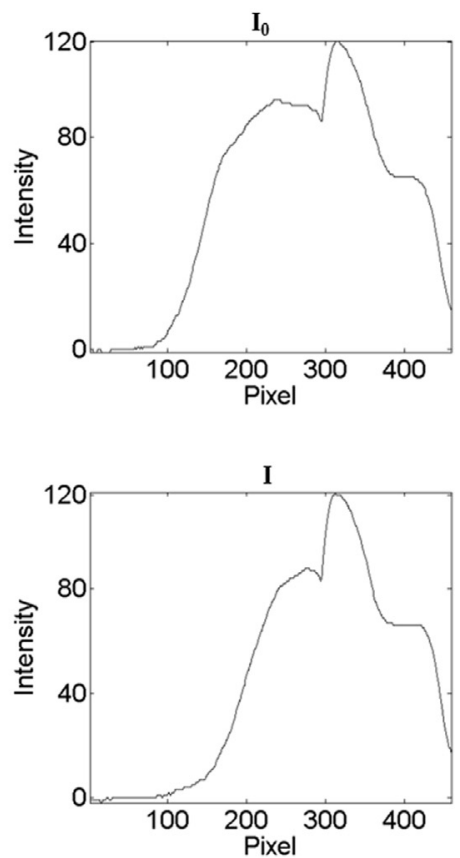

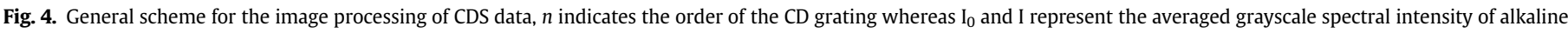
picrate solution and creatinine sample, respectively. 
paper. The spectral resolution along the horizontal axis of the ROI was determined to be $0.517 \mathrm{~nm} /$ pixel.

In the case of CSPT data the video recorded by the smartphone during the illumination sequence was first converted into AVI format and uploaded in Matlab workspace. The video stream consisted of 350 frames (14 frames/second), each containing a $480 \times 640$ pixels RGB image. In order to synchronize the frame rate of the illumination sequence with the one from the smartphone the packets of 7 consecutive frames were averaged to form a 50 frames sequence. From the resulting video stream a ROI centered on each sample well spot was manually selected (Supplementary Fig. S1). In order to minimize non-spatial homogeneity of both screen and camera a ROI located on a close background area was also considered. The RGB intensity values of the pixels enclosed by each ROI were averaged and a spectral fingerprint was calculated for each sample by subtracting the RGB values of the sample spot from its complementary background. To get rid of the intensity fluctuations usually observed for computer screens Savitzky-Golay filter (17 points window, second order polynomial) was applied to smooth the recovered spectral fingerprints. The latter were composed of 50 illumination colors by 3 color channels per sample.

Both CDS and CSPT output data were analyzed with Partial Least Squares (PLS) to establish the regression model for determination of creatinine concentration. The choice of the data processing method was made to accommodate the presence of non-creatinine chromogens which may interfere with creatinine measurement. To avoid scaling issues CSPT variables were scaled by the inverse of their respective standard deviation prior analysis. The set of available samples was randomly split into a calibration set (around 2/3 of the samples) from which the model was built and a test set (around $1 / 3$ of the samples) used for validation. The model prediction accuracy was assessed from both mean relative error (MRE) and the root mean square error of prediction (RMSEP) calculated according to Eqs. (2) and (3), respectively.

$$
\begin{aligned}
& \operatorname{MRE}(\%)=\sum_{\mathrm{i}=1}^{\mathrm{n}}\left(\frac{\left|y_{i}^{\text {pred }}-y_{i}^{\text {real }}\right|}{y_{i}^{\text {real }}}\right) \times \frac{100}{n} \\
& \operatorname{RMSEP}=\sqrt{\frac{1}{n} \sum_{i}\left(y_{i}^{\text {pred }}-y_{i}^{\text {real }}\right)^{2}}
\end{aligned}
$$

where $n$ is the number of samples in the validation test set, $y_{i}^{\text {pred }}$ is the creatinine concentration predicted by the model and $y_{i}^{\text {real }}$ is the reference concentration determined by capillary electrophoresis. PLS modeling was performed in the Unscrambler 9.7 (CAMO, Norway) software.

\section{Results and discussion}

\subsection{Reaction time optimization}

Time interval required for maximum color development is an important parameter for the Jaffe reaction. Reaction time is normally influenced by the concentration of the alkaline picrate solution. However, the presence of non-creatinine chromogens in the sample, which also react with alkaline picrate, can significantly blur the color development. To reduce color misinterpretation it should be considered that interferents are more likely to react late in the reaction [34]. This situation can be spotted by the formation of a plateau in absorbance values measured after several minutes. For a reliable determination of the rate of color development, real urine sample was considered in the present study. The Jaffe reaction was carried on by mixing $1 \mathrm{~mL}$ of alkaline picrate solution with $100 \mu \mathrm{L}$ of a 12-fold diluted urine sample in a $1 \times 1 \times 3 \mathrm{~cm}$ glass cuvette. Absorbance spectrum was measured every minute by the CDSpectroscope during $20 \mathrm{~min}$. The change of absorbance measured at $500 \mathrm{~nm}$ versus reaction time is displayed in Fig. 5. As expected the absorbance plateau is observed at longer time scale, typically after 15 min of reaction. Reversely, a linear response is displayed over the first $5 \mathrm{~min}$ of reaction which corresponds to the optimal time for color development. Therefore, this time interval was considered for the determination of creatinine in both standard and real urine samples.

\subsection{Standard creatinine assay}

At the first stage the optical responses provided by the CDSpectroscope and the CSPT platform were investigated on a set of working standard solutions $(\mathrm{n}=15)$ in the range from $160 \mu \mathrm{mol} \mathrm{L}^{-1}$ to $1.6 \mathrm{mmol} \mathrm{L}^{-1}$. Fig. 6a shows typical absorbance spectra (5 spectra out of 15 are reported for clarity) measured by CDS in the range from 425 to $660 \mathrm{~nm}$. Spectra of standard creatinine are characterized by a broad absorption band centered at $500 \mathrm{~nm}$ which can be safely attributed to the orange-yellow Janovsky complex [35]. A red-shift of the maximum of absorbance is observed once creatinine concentration reaches the threshold of $0.98 \mathrm{mmol} \mathrm{L}^{-1}$. To enable pixel to wavelength conversion the spectrum of a mercury vapor fluorescent lamp (Fig. 6b) was acquired under the same conditions. The wavelength calibration was performed by correlating the pixel position and the wavelength of the main absorption bands at 436.6, 484.7, 544.5 and $611.6 \mathrm{~nm}$ using linear function fitting. The band at $544.5 \mathrm{~nm}$ was defined as the average between mercury (546.5 nm) and terbium (542.4 nm) contributions which cannot be discriminated here due to the low spectral resolution of the $C D$ reflective grating. The corresponding calibration curve (Fig. 6a - inset) reveals an excellent correlation between pixel and wavelength and was further considered for CDS experiments. Since the alignment between CDS and the digital camera is likely to be different from day to day, it is recommended to build a new wavelength calibration for each position of the digital camera prior sample analysis.

Spectral fingerprints of the 15 standard samples measured by the CSPT platform are displayed in Fig. 6c. Data reveal the presence of both positive and negative spectral contributions which can be directly attributed to the optical properties of the sample. Thus,

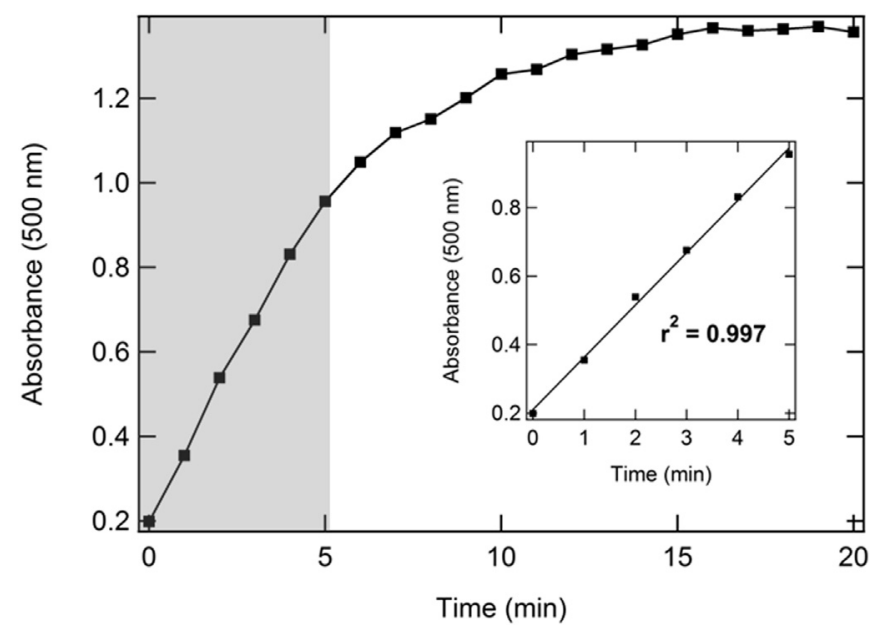

Fig. 5. Change of absorbance of urinary creatinine measured as function of time by the proposed CD-Spectroscope based on Jaffe reaction, the linear range is highlighted by the gray box. 

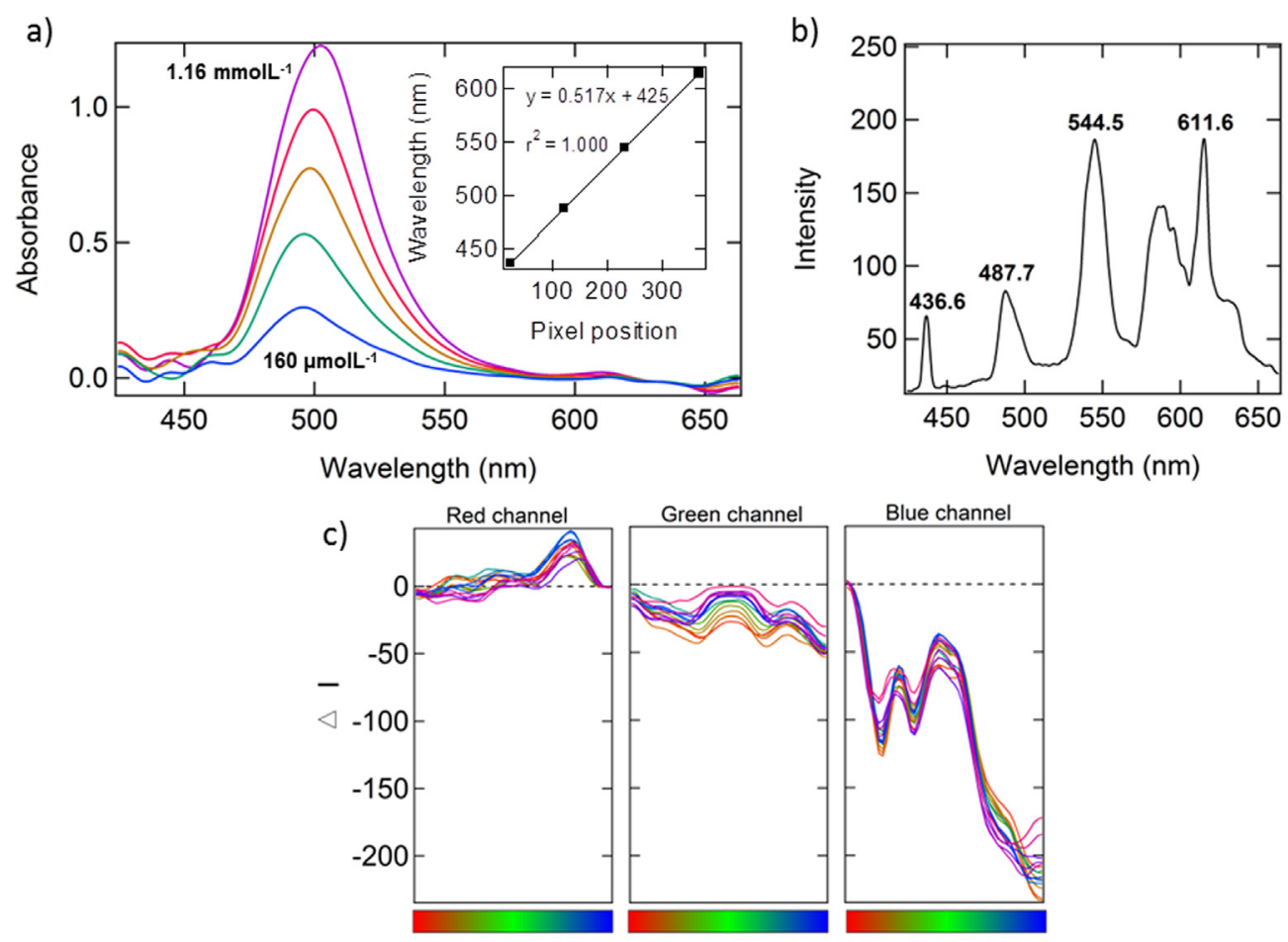

Illumination Sequence

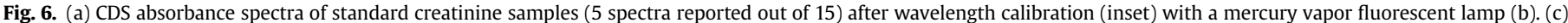
CSPT fingerprints of all 15 samples following background subtraction.

negative signal corresponds to absorbance since the light transmitted through the sample is weaker than the background, while positive signal can be ascribed to emission as the sample fluorescence is excited by the screen light. The results show strong absorbance for blueish illuminations which is consistent with the orange-yellow color of the sample. In the same way the significant absorbance for greenish illuminations is in agreement with the Janovsky complex band located at $500 \mathrm{~nm}$. Interestingly, emission is directly observed on the red channel and is found in situation where the red intensity of the sample is lower comparing to the blue channel.

In order to estimate the concentration of creatinine from both CDS and CSPT data partial least squares (PLS) approach was employed. Data sets were randomly split in two parts used for training and test set. A set of 5 samples was considered for the test validation whereas the remaining 10 samples were used for calibration. The scattered plots representing the measured versus predicted creatinine concentration calculated from the validation set are shown Fig. 7a and $b$ for CDS and CSPT data, respectively. The performance of the regression models evaluated through MRE and RMSEP is given in Table 1. Scattered plots and performance metrics for training data sets are available in Supplementary data (Fig. S2 and Table S1).

Both models exhibited good linearity between measured and predicted creatinine concentrations with $\mathrm{r}^{2}$ values above 0.99 and MRE lower than $10 \%$ which is the average tolerance for the Jaffe reaction in clinical analysis [36]. To evaluate the compatibility of the present approach with other devices the same sample array was analyzed with another set of digital camera (Samsung ST65-14.2 M pixels) and smartphone (Huawei H60-L04 - $13 \mathrm{M}$ pixels). Results show that the MRE does not exceed $7.8 \%$ which is consistent with the values reported in Table 1 . Since the number of samples was not large, a $k$-fold random split validation test was performed to confirm the robustness of the model. The random split of the samples into calibration $(n=10)$ and validation $(n=5)$ set was performed 20 times and for each run the MRE and RMSEP were calculated. The average MRE was found to be in the range from 8.6 to $9.0 \%$ whereas the mean RMSEP is ranging from 0.060 to $0.068 \mathrm{mmol} \mathrm{L}^{-1}$. These results suggest that the performance of CDS and CSPT platforms is appropriate for creatinine quantification.

For CDS data the choice of three latent variables in PLS model is justified by the presence of a red-shift of the maximum of absorbance when concentration rises from $160 \mu \mathrm{mol} \mathrm{L}^{-1}$ to $1.6 \mathrm{mmol} \mathrm{L}^{-1}$ (Fig. 6a). The limits of detection (LOD) were evaluated from the IUPAC consistent approach recently proposed by Allegrini and Olivieri [37] and were estimated to be in the range $72-89 \mu \mathrm{mol} \mathrm{L}^{-1}$ for the CDS platform and $92-111 \mu \mathrm{mol} \mathrm{L}^{-1}$ for the CSPT platform. Additionally, the repeatability of both techniques was investigated from the comparison of the PLS models calculated on three consecutive days. Coefficients of variations show a good inter-day repeatability of $3.6 \%$ and $6.5 \%$ for CDS and CSPT platforms respectively. Similar conclusion can be reported for the measurement of $0.95 \mathrm{mmol} \mathrm{L}^{-1}$ creatinine $(\mathrm{n}=6)$ with intra-day coefficients of variation of $1.7 \%$ (CDS) and $2.9 \%$ (CSPT). The absence of significant differences in the quantitative analysis of standard creatinine samples from day to day suggests that the signal have a low sensitivity to experimental conditions. These observations indicate that the two developed platforms are indeed appropriate for the analysis of creatinine.

\subsection{Quantification of urinary creatinine}

The developed CDS and CSPT platforms were applied for the determination of creatinine in real human urine samples. Absorption spectra and spectral fingerprints collected for the 26 samples are shown in Supplementary data (Fig. S3 and S4). The data set was 
a)

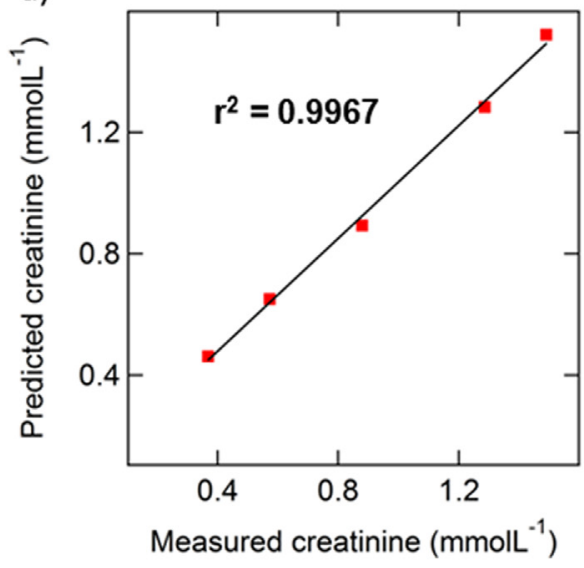

b)

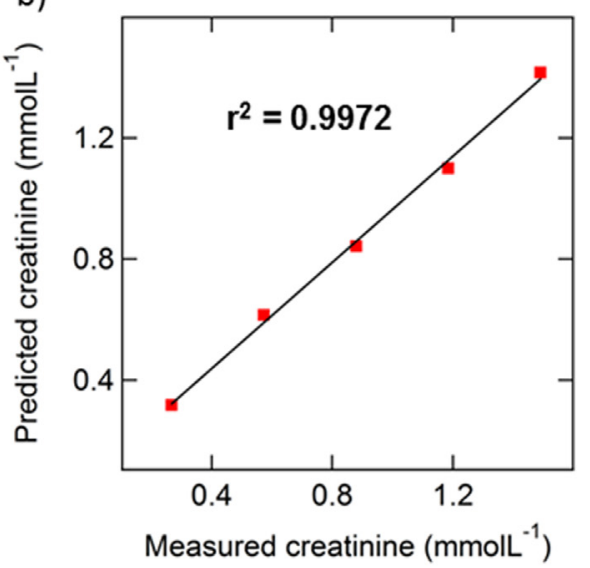

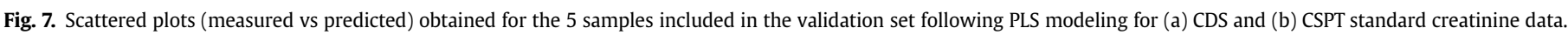

Table 1

Prediction performance of the two PLS models in the validation test set $(n=5)$ for CDS and CSPT standard creatinine data.

\begin{tabular}{lllll}
\hline Input data & Latent variables & $\operatorname{RMSEP}\left(\mathrm{mmol} \mathrm{L}^{-1}\right)$ & $\mathrm{MRE}(\%)$ & $\mathrm{r}^{2}$ \\
\hline CDS & 3 & 0.056 & 8.2 & 0.9967 \\
CSPT & 1 & 0.060 & 8.5 & 0.9972 \\
\hline
\end{tabular}

randomly split in a training set of 18 samples and validation set of 8 samples. Scattered plots of the validation test set for CDS and CSPT platforms are shown in Fig. $8 \mathrm{a}$ and b, respectively. The performance of the regression models is resumed in Table 2.

Fig. 8 shows that a significant linear correlation $\left(r^{2}>0.93\right)$ can be found between urinary creatinine concentrations predicted by the PLS model and reference values measured by capillary electrophoresis. The mean relative error remains below the $10 \%$ mark which suggests that the two proposed approaches have a low sensitivity to the presence of non-creatinine chromogens in human urine. This observation is consistent with the 20 -fold random validation test for which average MRE of $9.8 \%$ and $9.4 \%$ have been found for CDS and CSPT data respectively. The increase in MRE comparing to the values listed in Table 2, especially for CSPT data, is a clear indicator of non-creatinine chromogens affecting the predictive accuracy of the model. This is particularly true once considering 20-splits averaged RMSEP values for CDS
Table 2

Performances of the two PLS models applied on the validation test set $(n=8)$ for CDS and CSPT urinary creatinine data.

\begin{tabular}{lllll}
\hline Input data & Latent variables & RMSEP $\left(\mathrm{mmol} \mathrm{L}^{-1}\right)$ & MRE $(\%)$ & $\mathrm{r}^{2}$ \\
\hline CDS & 1 & 0.057 & 8.4 & 0.9303 \\
CSPT & 2 & 0.033 & 6.7 & 0.9771 \\
\hline
\end{tabular}

(0.071 $\left.\mathrm{mmol} \mathrm{L}^{-1}\right)$ and CSPT $\left(0.064 \mathrm{mmol} \mathrm{L}^{-1}\right)$ data.

In the case of CDS data a decrease of the number of PLS latent variables comparing with standard samples can be considered surprising at the first sight. A plausible explanation is that the concentration range of urinary samples does not exceed $0.98 \mathrm{mmol} \mathrm{L}^{-1}$ which was found to be the threshold beyond which a red-shift of the maximum of absorbance was observed (Fig. 6a). This can be confirmed by a detailed examination of urinary creatinine spectra (Fig. S3).

\subsection{Comparison between CDS and CSPT systems}

In clinical diagnostics high throughput is normally required as few tens to several hundred samples should be processed per day. In this regard the CSPT platform is a more appropriate system with a maximum rate of analysis of 96 samples in $25 \mathrm{~s}$ while the $\mathrm{CD}$ Spectroscope measurement require several hours for the same
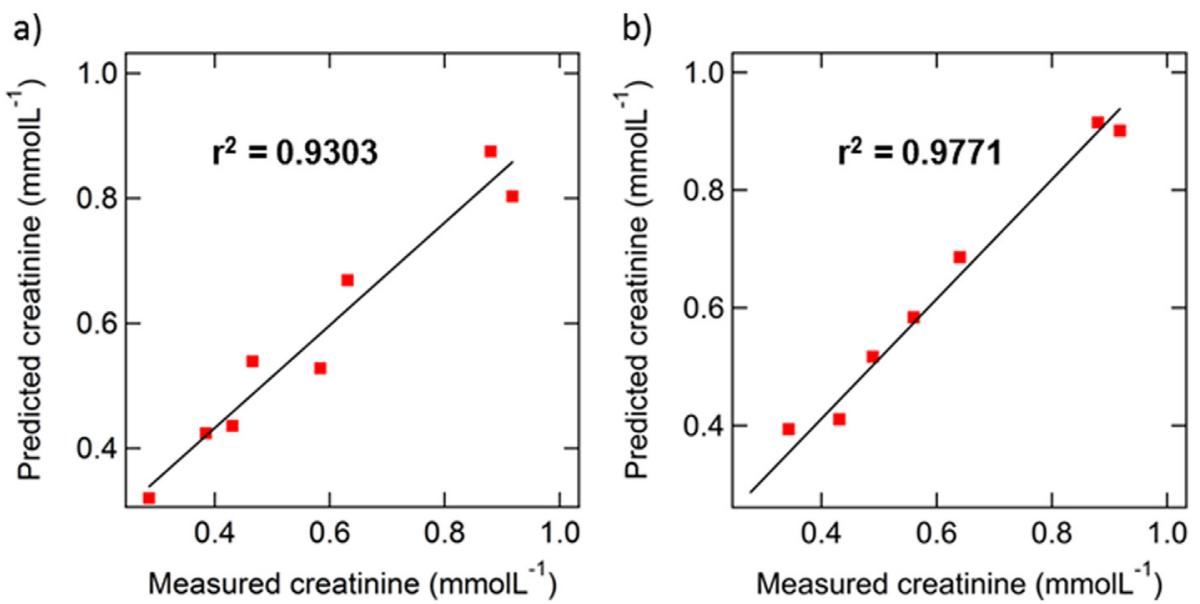

Fig. 8. Measured vs predicted plots for PLS regression model validation with 8 samples for (a) CDS and (b) CSPT urinary creatinine data. 
number of samples. On the other hand, the CD-Spectroscope is more compact, portable and does not necessary require power supply as any other available light source can be used. This latter advantage makes CDS highly attractive platform with potential for on-site measurements in distant locations without access to professional labs.

Regarding the analytical performance of the developed platforms in determination of urinary creatinine, the lowest RMSEP was achieved for CSPT data. This might be attributed to the fact that the sample position is not perfectly reproducible in CDS experiments and mainly depends on the user. However, the prediction accuracy of both PLS models built from CDS and CSPT data is within the $10 \%$ standard error routinely tolerated for the Jaffe kinetic reaction. Finally, both systems achieved detection limits (89-111 $\mu \mathrm{mol} \mathrm{L}^{-1}$ ) which are acceptable for clinical diagnostics as similar order of magnitude was reported in the literature for other creatinine assays $[3,7,10]$.

\section{Conclusion}

Two cost-effective home-made platforms based on CDSpectroscope and CSPT illumination sequence have been developed for the quantitative determination of creatinine in human urine. Optical properties of the 26 urine samples were captured by either a digital camera or a smartphone video stream and converted into absorbance spectra and spectral fingerprints respectively. These data were used as inputs for PLS regression modeling to predict creatinine content using capillary electrophoresis data as reference. The results suggest that the developed platforms for the quantification of urinary creatinine are meeting the requirements for clinical analysis. Both systems were found to achieve similar performance in terms of accuracy with a mean relative error in creatinine determination below the $10 \%$ mark, which is normally considered for routine clinical analysis based on the Jaffe reaction. Even if the proposed platforms can hardly compete with sophisticated techniques like e.g. HPLC, they still can provide for reliable quantitative analysis of creatinine level in human urine.

\section{Acknowledgments}

B. Debus would like to acknowledge the financial support from St. Petersburg State University PostDoc Grant \#12.50.1191.2014. This work was partially financially supported by Government of Russian Federation, Grant 074-U01.

\section{Appendix A. Supplementary data}

Supplementary data related to this article can be found at http:// dx.doi.org/10.1016/j.aca.2015.09.007.

\section{References}

[1] K. Spencer, Analytical reviews in clinical biochemistry: the estimation of creatinine, Ann. Clin. Biochem. 23 (1986) 1-25.

[2] W.R. De Araújo, M.O. Salles, T.R.L.C. Paixão, Development of an enzymeless electroanalytical method for the indirect detection of creatinine in urine samples, Sens. Actuators B Chem. 173 (2012) 847-851.

[3] E.M.K. Leung, W. Chan, A novel reversed-phase HPLC method for the determination of urinary creatinine by pre-column derivatization with ethyl chloroformate: comparative studies with the standard Jaffé and isotopedilution mass spectrometric assays, Anal. Bioanal. Chem. 406 (2014) 1807-1812.

[4] K. Nakamoto, R. Kurita, O. Niwa, One-chip biosensor for simultaneous disease marker/calibration substance measurement in human urine by electrochemical surface plasmon resonance method, Biosens. Bioelectron. 26 (2010) 1536-1542.

[5] C.H. Nieh, S. Tsujimura, O. Shirai, K. Kano, Amperometric biosensor based on reductive $\mathrm{H}_{2} \mathrm{O}_{2}$ detection using pentacyanoferrate-bound polymer for creatinine determination, Anal. Chim. Acta 767 (2013) 128-133.

[6] E.P. Randviir, D.K. Kampouris, C.E. Banks, An improved electrochemical creatinine detection method via a Jaffe-based procedure, Analyst 138 (2013) 6565-6572.

[7] P.C. Pandey, A.P. Mishra, Novel potentiometric sensing of creatinine, Sens. Actuators B Chem. 99 (2004) 230-235.

[8] R.S. Carling, S.L. Hogg, T.C. Wood, J. Calvin, Simultaneous determination of guanidinoacetate, creatine and creatinine in urine and plasma by underivatized liquid chromatography-tandem mass spectrometry, Ann. Clin. Biochem. 45 (2008) 575-584.

[9] X. Li, X. Fang, Z. Yu, G. Sheng, M. Wu, J. Fu, H. Chen, Direct quantification of creatinine in human urine by using isotope dilution extractive electrospray ionization tandem mass spectrometry, Anal. Chim. Acta 748 (2012) 53-57.

[10] M. Niesser, B. Koletzko, W. Peissner, Determination of creatinine in human urine with flow injection tandem mass spectrometry, Ann. Nutr. Metab. 61 (2012) 314-321.

[11] S. Fraselle, K. De Cremer, W. Coucke, G. Glorieux, J. Vanmassenhove, E. Schepers, N. Neirynck, I. Van Overmeire, J. Van Loco, W. Van Biesen, R. Vanholder, Development and validation of an ultra-high performance liquid chromatography-tandem mass spectrometry method to measure creatinine in human urine, J. Chromatogr. B Anal. Technol. Biomed. Life Sci. 988 (2015) 88-97.

[12] S. Wang, X. Li, J. Yang, X. Yang, F. Hou, Z. Chen, Rapid determination of creatinine in human urine by microchip electrophoresis with LED induced fluorescence detection, Chromatographia 75 (2012) 1287-1293.

[13] V. Pavlíček, P. Tủma, J. Matějčková, E. Samcová, Very fast electrophoretic determination of creatinine and uric acid in human urine using a combination of two capillaries with different internal diameters, Electrophoresis 35 (2014) 956-961.

[14] R. Gatti, V. Lazzarotto, C.B. De Palo, E. Cappellin, P. Spinella, E.F. De Palo, A rapid urine creatinine assay by capillary zone electrophoresis, Electrophoresis 20 (1999) 2917-2921.

[15] T.T. Zava, S. Kapur, D.T. Zava, Iodine and creatinine testing in urine dried on filter paper, Anal. Chim. Acta 764 (2013) 64-69.

[16] K.K. Fung, C.P. Chan, R. Renneberg, Development of a creatinine enzymebased bar-code-style lateral-flow assay, Anal. Bioanal. Chem. 393 (2009) $1281-1287$.

[17] R. Stosch, A. Henrion, D. Schiel, B. Güttler, Surface-enhanced Raman scattering based approach for quantitative determination of creatinine in human serum, Anal. Chem. 77 (2005) 7386-7392.

[18] J. Vasiliades, Reaction of alkaline sodium picrate with creatinine: I. Kinetics and mechanism of formation of the mono creatinine picric acid complex, Clin. Chem. 22 (1976) 1664-1671.

[19] R.W. Bonsnes, H.H. Taussky, On the colorimetric determination of creatinine by the Jaffe reaction, J. Biol. Chem. 158 (1945) 581-591.

[20] W.T. Caraway, C.W. Kammeyer, Chemical interference by drugs and other substances with clinical laboratory test procedures, Clin. Chim. Acta 41 (1972) 395-434.

[21] K. Larsen, Creatinine assay by a reaction-kinetic principle, Clin. Chim. Acta 41 (1972) 209-217.

[22] D.S. Lee, B.G. Jeon, C. Ihm, J.K. Park, M.Y. Jung, A simple and smart telemedicine device for developing regions: a pocket-sized colorimetric reader, Lab Chip 11 (2011) 120-126.

[23] L. Shen, J.A. Hagen, I. Papautsky, Point-of-care colorimetric detection with a smartphone, Lab Chip 12 (2012) 4240-4243.

[24] J.I. Hong, B.Y. Chang, Development of the smartphone-based colorimetry for multi-analyte sensing arrays, Lab Chip 14 (2014) 1725-1732.

[25] S. Lee, V. Oncescu, M. Mancuso, S. Mehta, D. Erickson, A smartphone platform for the quantification of vitamin D levels, Lab Chip 14 (2014) 1437-1442.

[26] C.P. Gulka, J.D. Swartz, D.W. Wright, Ni(II)NTA AuNPs as a low-resource malarial diagnostic platform for the rapid colorimetric detection of Plasmodium falciparum histidine-rich protein-2, Talanta 135 (2015) 94-101.

[27] V. Oncescu, D. O'Dell, D. Erickson, Smartphone based health accessory for colorimetric detection of biomarkers in sweat and saliva, Lab Chip 13 (2013) $3232-3238$

[28] K.D. Long, H. Yu, B.T. Cunningham, Smartphone instrument for portable enzyme- linked immunosorbent assays, Biomed. Opt. Express 5 (2014) 3792-3806

[29] A.W. Martinez, S.T. Phillips, E. Carrilho, S.W. Thomas Iii, H. Sindi, G.M. Whitesides, Simple telemedicine for developing regions: camera phones and paper-based microfluidic devices for real-time, off-site diagnosis, Anal. Chem. 80 (2008) 3699-3707.

[30] D. Filippini, J. Bakker, I. Lundstrom, Fingerprinting of fluorescent substances for diagnostic purposes using computer screen illumination, Sens. Actuators B Chem. 106 (2005) 302-310.

[31] S. Wold, M. Sjöström, L. Eriksson, PLS-regression: a basic tool of chemometrics, Chemom. Intell. Lab. Syst. 58 (2001) 109-130.

[32] A.A. Sidorova, A.V. Grigoriev, Determination of diagnostical markers of urolithiasis by capillary electrophoresis, J. Anal. Chem. 67 (2012) 478-485.

[33] F. Wakabayashi, Resolving spectral lines with a periscope-type DVD spectroscope, J. Chem. Educ. 85 (2008) 849-853.

[34] T. Songjaroen, T. Maturos, A. Sappat, A. Tuantranont, W. Laiwattanapaisal, 
Portable microfluidic system for determination of urinary creatinine, Anal. Chim. Acta 647 (2009) 78-83.

[35] L.D. Bowers, E.T. Wong, Kinetic serum creatinine assays. II. A critical evaluation and review, Clin. Chem. 26 (1980) 555-561.

[36] M.V. Guterres, P.O.L. Volpe, M.M.C. Ferrerira, Multiway calibration for creatinine determination in human serum using the Jaffe reaction, Appl. Spectrosc. 58 (2004), 256-256.

[37] F. Allegrini, A.C. Olivieri, IUPAC-consistent approach to the limit of detection in partial least-squares calibration, Anal. Chem. 86 (2014) 7858-7866. 